\title{
Clathrate formation and dissociation in vapour/water/ice/hydrate systems in SBA-15, Sol-Gel and CPG porous media, as probed by NMR relaxation, novel protocol NMR Cryoporometry, Neutron Scattering and ab-initio QM-MD simulation.
}

\author{
J. Beau W. Webber a,b,* Ross Anderson ${ }^{\mathrm{a}}$ John H. Strange ${ }^{\mathrm{b}}$ Bahman Tohidi ${ }^{\mathrm{a}}$ \\ a Institute of Petroleum Engineering, Heriot-Watt University, Edinburgh. EH14 4 AS \\ ${ }^{\mathrm{b}}$ School of Physical Sciences, University of Kent, Canterbury, Kent. CT2 7NH
}

\begin{abstract}
The Gibbs Thomson effect modifies the pressure and temperature at which clathrates occur, hence altering the depth at which they occur in the sea-bed. NMR measurements as a function of temperature are being conducted for water/ice/hydrate systems in a range of pore geometries, including templated SBA-15 silicas, controlled pore glasses and sol-gel silicas. Rotator-phase plastic ice is shown to be present in confined geometry, and bulk tetrahydrofuran hydrate is also shown to probably have a rotator phase. A novel NMR Cryoporometry protocol has been developed that probes both the melting and freezing events, while avoiding the usual problem of supercooling for the freezing event. This enables a detailed probing of the system for a given pore size and geometry, and exploration of the differences between the hydrate formation and dissociation processes inside the pores. These process differences have an important effect for the environment, as they impact on the ability of a marine hydrate system to re-form once warmed above a critical temperature. Ab-Initio Quantum-Mechanical Molecular-Dynamics calculations are also being employed to probe the dynamics of liquids in pores at nanometric dimensions.
\end{abstract}

Key words:

clathrate, hydrate, confined geometry, NMR, nmr relaxation, cryoporometry, neutron scattering, PVT, ab-initio, molecular-dynamics, plastic ice, global warming

PACS: 64.60.-i, 65.60.+a, 81.07.-b, 03.75.Hh, 05.70.Fh, 64.60.Qb, 64.70.Dv, 68.03.Cd, 68.08.-p, 82.56.Na, 82.56.Ub, 61.43.Fs, 61.43.Gt, $61.46 .+\mathrm{w}, 82.60 . \mathrm{Qr}$

\section{Introduction.}

There is currently great interest in naturally occurring methane gas hydrates (figure 1a) as strategic energy reserve and also in the possibilities for $\mathrm{CO}_{2}$ disposal in the sea-bed (as hydrate and as liquid). The processes driving clathrate formation and dissociation in

\footnotetext{
* Correspondence address: Beau Webber, Lab-Tools Ltd., Canterbury Enterprise Hub, University of Kent, Canterbury, Kent. CT2 7NJ. +44 (0) 1227824675

Email address: J.B.W.Webber@kent.ac.uk (J. Beau W. Webber).

URL: http://www.Lab-Tools.com (J. Beau W. Webber).
}

porous media such as marine sediments are controlled by thermodynamics, wetting, diffusion and the percolation of gas and water (1). We are studying these processes by PVT measurements, by wide-temperaturerange NMR relaxation, by NMR Cryoporometry using a novel protocol, by Neutron Diffraction, and by Small Angle Neutron Scattering (SANS).

For marine hydrates the phase diagram for methane hydrate may be re-mapped to obtain a diagram related to depth below the sea-surface - this indicates the expected hydrate stability zone (figure $1 \mathrm{~b}$ ). However, the sea bed is a porous medium, and the Gibbs equations show that it is reasonable to expect a change to the 
thickness of this zone of hydrate stability, due to confining geometry. We report on preliminary measurements studying tetrahydrofuran (THF) liquid hydrates at ambient pressure, as a function of temperature, comparing the behaviour of bulk hydrate with that confined in Controlled Pore Glass (CPG). Comparison is also made between pure water/ice systems in confined geometry, and hydrate systems in confined geometry.
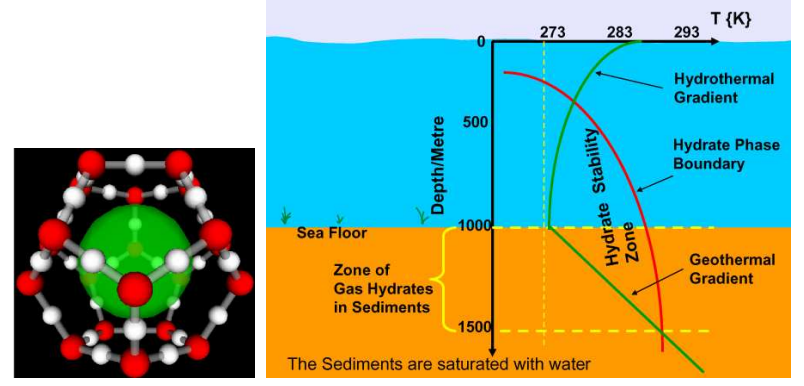

Fig. 1. a) Hydrate water cage containing gas. b) Marine hydrate phase diagram remapped as a function of depth, showing the expected hydrate stability zone.

\section{2. $\mathbf{T}_{2}$ transverse relaxation times for pure water/ice in confined geometry.}

The NMR relaxation behaviour observed for the water/ice in SBA-15 porous silica is dependent on temperature, and as well as 'water-like' and 'ice-like' phases, a phase with intermediate properties is observed. Pure bulk water has a $T_{2}$ relaxation time of the order of a few seconds at the $0.5 \mathrm{~T} \mathrm{~B}_{0}$ static magnetic field used for this relaxation work. However, in the pores of this SBA-15 silica, the surface relaxivity reduces the $T_{2}$ of the water to about $8 \mathrm{~ms}$. In comparison, the bulk phase of brittle ice has an approximately Gaussian free induction decay with a relaxation time of the order of $10 \mu \mathrm{s}$.

The free induction decay (FID) for this sample at $\sim 190 \mathrm{~K}$ is very similar to that of bulk ice, but as the temperature is raised by $20 \mathrm{~K}$ to $210 \mathrm{~K}$ some of the brittle ice transforms to a component with a longer relaxation time and with a form that becomes increasingly exponential. Measurement of the variation of the $\mathrm{T}_{2}$ relaxation rate with temperature for this longer relaxation component, as measured by Carr-PurcellMeiboom-Gill (CPMG) and $T_{2}{ }^{*}$ corrected FID, are plotted in Fig. 2. Above $220 \mathrm{~K}$, the shorter brittle ice component reduces in amplitude as it converts to the longer form, while the longer component of the FID remains fairly constant in relaxation time, between 100 to $200 \mu \mathrm{s}$, and its amplitude progressively increases with temperature. Just below the melting temperature of the ice in the pores, at the Gibb-Thomson lowered temperature of about $260 \mathrm{~K}$, there is a rapid increase in the relaxation time of the longer component. Neutron diffraction measurements show a similar component, as a broad peak characteristic of defective ice/water.

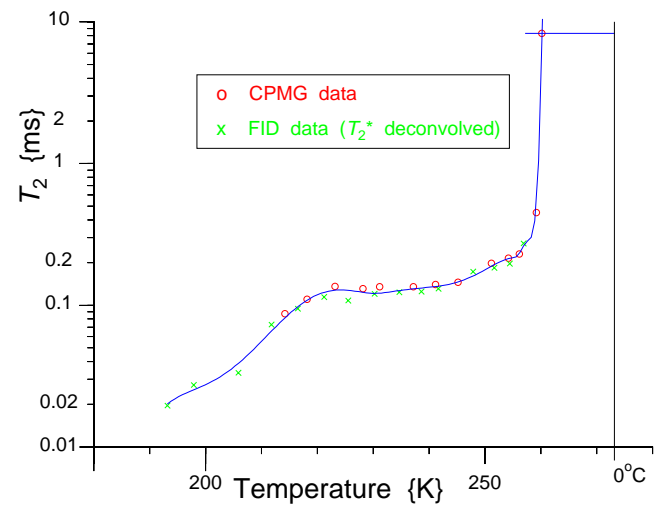

Fig. 2. Variation with temperature of the long $T_{2}$ relaxation time component for plastic ice in SBA-15 porous silica.

There are three possible causes that need to be considered for this factor of 10 increase in the $\mathrm{T}_{2}$ at around $200 \mathrm{~K}$ to $220 \mathrm{~K}$, for the defective ice, over that for brittle ice; these are discussed in detail elsewhere (2).

However it can be seen that the plot of $\mathrm{T}_{2}(T)$ in figure 2 is very similar to that for the plastic phases of materials such as cyclohexane, and it is believed that the same processes are involved in both cases. Thus the rapid rise in the 200 to $180 \mathrm{~K}$ is due to the onset of rotational motion, well known to give rise to a form of motional averaging such that the actual proton separations must be replaced by the separation of the molecular centres. This gives rise to a significant increase in $\mathrm{T}_{2}$ at the onset of rotational motion, followed by a plateau in $\mathrm{T}_{2}(T)$ as $\mathrm{T}$ increases (3). Translational motion sets in just prior to the melting event.

Consequently it is suggested that this longer ice component is in a plastic crystalline state and that this plastic ice corresponds to the disordered component as measured by neutron scattering. These NMR measurements will be reported in detail in a separate paper (4); combined with the neutron data, they indicate that there is an equilibrium state within the interfacial layer in the pores, such that the ice changes continuously and reversibly with temperature between a brittle cubic crystalline phase (presumably located at the pore centre) and a plastic disordered rotator phase (presumably mostly located near the silica pore surface).

\section{3. $\mathbf{T}_{2}$ relaxation times and signal amplitudes for bulk THF hydrate.}

The bulk tetrahydrofuran shows both brittle ice-like and longer exponential relaxation $T_{2}$ components, converting to mostly brittle ice at around $220 \mathrm{~K}$. As the 
temperature is raised the amplitude of the longer component increases, and in the $20 \mathrm{~K}$ below the dissociation temperature the $T_{2}$ increases to around 100 to $200 \mu \mathrm{s}$, comparable with that for the plastic ice in confined geometry. Thus, although the plateau in relaxation time is not so clear as for the case of pure water in confined geometry, there is a suggestion that in this region the THF hydrate may be in a rotationally mobile state, with again translational motion becoming significant just prior to the dissociation event.

THF hydrate long-component $T_{2}$
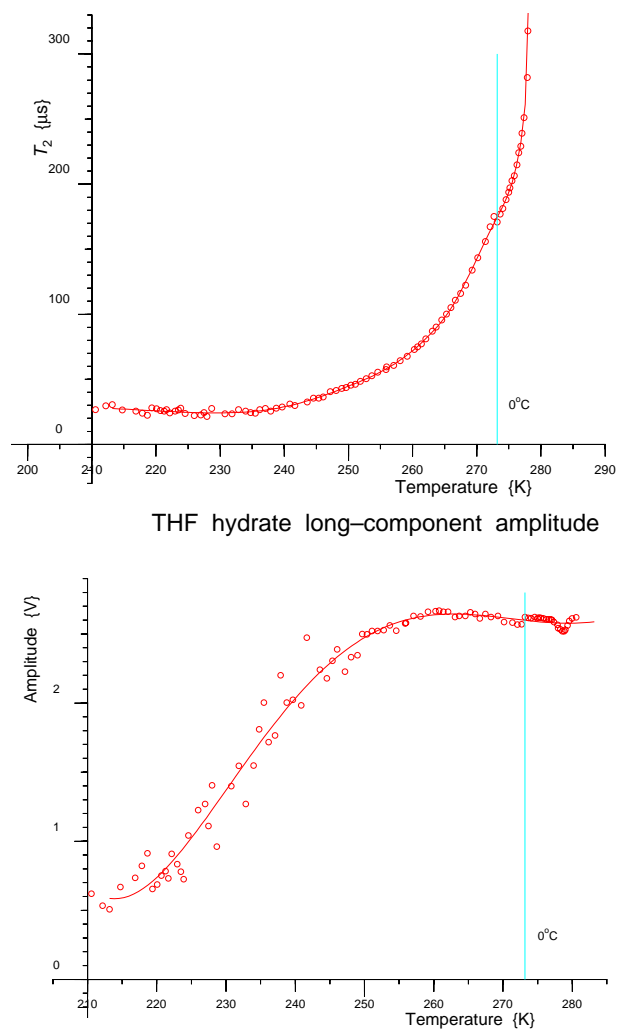

Fig. 3. Variation with temperature of a) the $T_{2}$ of the long $T_{2}$ relaxation time component, b) the amplitude of this component, for bulk THF hydrate.

\section{Novel multi-ramp NMR Cryoporometry measurements for both pure water and THF hydrate in confined geometry.}

Liquids in confined geometry exhibit equilibrium melting and freezing point depressions, $\Delta \mathrm{T}$, compared with the normal bulk melting point, as described by the Gibbs-Thomson relation, which may be written simply as (5):-

$$
\Delta T=\frac{K_{G T}}{x}
$$

where $K_{G T}$ is a constant that depends on the liquid, the crystal/liquid interface geometry and the wetting nature of the pore walls. $x$ is a dimension that defines the effective size of the pore (6). The technique is analogous to differential scanning calorimetry, but has advantages that have been discussed previously $(7 ; 8)$.

NMR Cryoporometry measurements have conventionally been performed using a warming temperature ramp. This is because, on a cooling ramp, liquids such as water in porous media frequently exhibit a metastable supercooling of typically 15 to $20 \mathrm{~K}$, thus often obscuring the equilibrium Gibbs-Thomson freezing transition.

A novel multi-ramp Cryoporometry protocol has been developed (figure 4), (that can also be used for DSC), where the sample is initially cooled through the supercooling event until all the liquid is frozen, then warmed until the liquid in the pores melts but the liquid around the grains remains frozen. During a subsequent cooling ramp, the ice around the grains serves to initiate freezing inside the pores at the true Gibbs-Thomson equilibrium freezing point. A final warming ramp again melts the liquid in the pores, and then finally the liquid around the grains. The latter event serves as a reference temperature for the bulk melting point, helping remove instrumentation offsets.

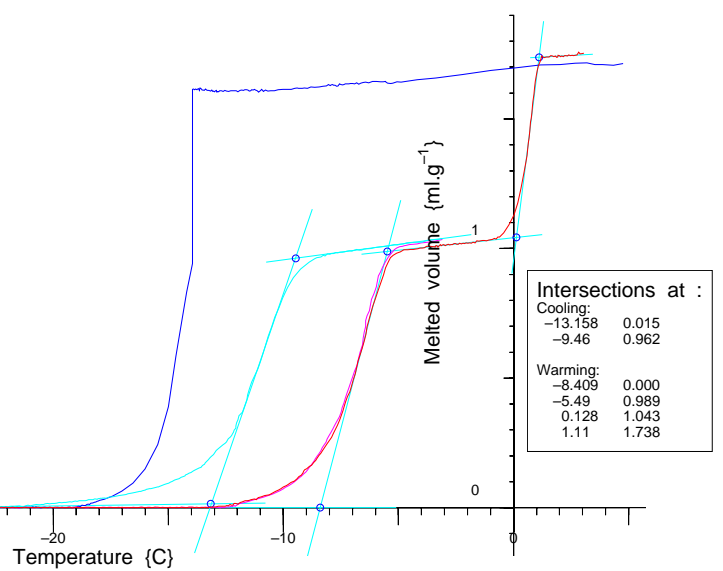

Fig. 4. Novel multi-ramp NMR Cryoporometry measurements on water in $10 \mathrm{~nm}$ pore diameter sol-gel silica, probing freezing and melting events.

Applying this novel protocol to THF hydrate in CPG silica, (figure 5), the initial $20 \mathrm{~K}$ supercooling (not shown) now no longer obscures the equilibrium hydrate formation event in the pores. This measurement shows two important features : 1) the hydrate dissociation temperature in the pores is lowered by about $2 \mathrm{~K}$ - not a great amount, but this is still sufficient to correspond to a change of marine hydrate layer thickness of about $60 \mathrm{~m} ; 2)$ of greater significance, the hydrate formation temperature is lowered by about $7.4 \mathrm{~K}$. 
The environmental impact of this difference between hydrate formation and dissociation temperatures, is that for a marine hydrate, a small rise in sea temperature (say $0.1 \mathrm{~K}$ ), causing the dissociation of some hydrate and the release of some methane gas, is not reversible by an equivalent drop in sea temperature, but instead requires a far greater temperature decrease before the hydrate will re-form.

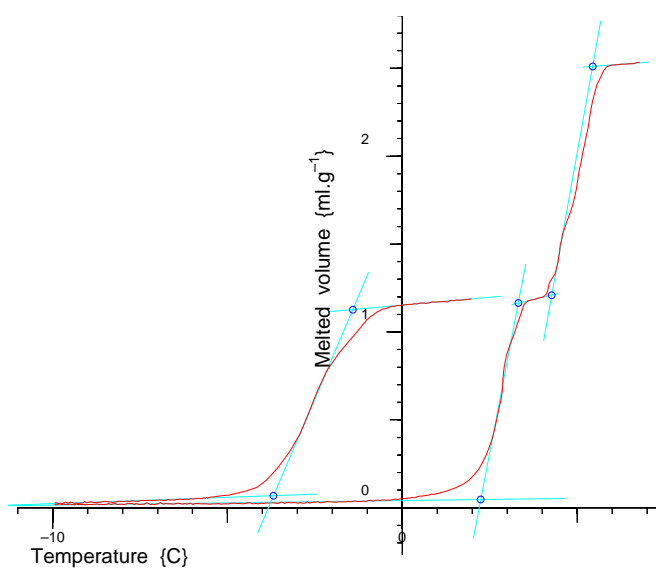

Fig. 5. Novel multi-ramp NMR Cryoporometry measurements on THF hydrate in $30 \mathrm{~nm}$ pore diameter CPG porous silica, probing the hydrate formation event as well as dissociation events.

\section{Current and future work.}

A high pressure NMR probe has been designed for NMR relaxation and Cryoporometry on gas hydrates, as a function of pressure, temperature and gas loading.

Ab-Initio Quantum-Mechanical Molecular-Dynamics studies are being conducted of liquid and gas molecules in model porous systems (figure 6), and studies on hydrate systems will be initiated.

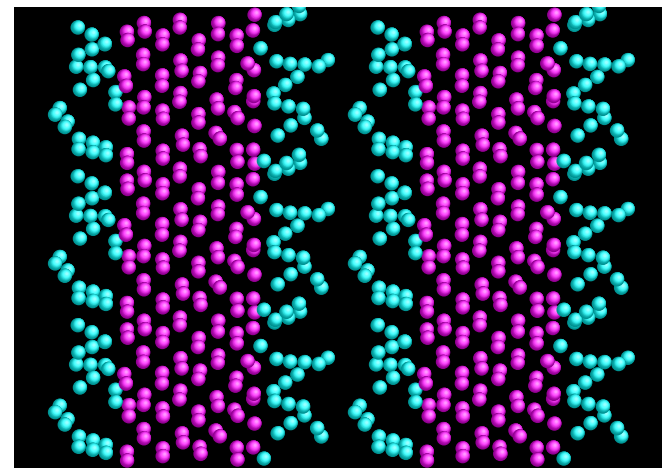

Fig. 6. One time-step from a CASTEP ab-initio Quantum-Mechanical Molecular-Dynamical simulation, for a porous system constructed from infinite laminar planes of silicon, with nitrogen introduced into the void space.

\section{Acknowledgments}

This study is supported by EPSRC grant EP/D052556/1: Capillary controls on gas hydrate growth and dissociation in synthetic and natural porous media: PVT, NMR, Neutron Diffraction and SANS.

\section{References}

[1] K. Ostergaard, R. Anderson, M. Llamedo, B. Tohidi, Hydrate phase equilibria in porous media: effect of pore size and salinity, Terra Nova 14 (5) (2002) 307-312.

[2] E. Liu, J. C. Dore, J. B. W. Webber, D. Khushalani, S. Jähnert, G. H. Findenegg, T. Hansen, Neutron diffraction and $\mathrm{nmr}$ relaxation studies of structural variation and phase transformations for water/ice in sba-15 silica: I. the over-filled case, Journal of Physics: Condensed Matter 18 (44) (2006) 10009-10028.

URL http: //stacks . iop.org/0953-8984/18/10009

[3] J. Chezeau, J. Strange, Diffusion in molecularcrystals, Physics Reports - Review Section of Physics Letters 53 (1) (1979) 1-92.

[4] J. Webber, J. Strange, in preparation.

[5] J. Strange, M. Rahman, E. Smith, Characterisation of porous solids by nmr, Phys. Rev. Letts. 71 (21) (1993) 3589-3591.

[6] J. Webber, A generalisation of the thermoporisimetry gibbs-thomson equation for arbitrary pore geometry, Magn. Reson. Imaging 21 (3-4) (2003) 428. URL http: //dx.doi .org/10.1016/S0730-725X (03) 00172-3

[7] J. Webber, J. Strange, J. Dore, An evaluation of nmr cryoporometry, density measurement and neutron scattering methods of pore characterisation, Mag. Res. Imag. 19 (3-4) (2001) 395-399.

[8] B. Webber, J. Dore, Structural and dynamic studies of water in mesoporous silicas using neutron scattering and nuclear magnetic resonance, Journal of Physics: Condensed Matter 16 (Special Issue: Water in Confined Geometry) (2004) S5449-S5470. URL http: //stacks . iop. org/JPhysCM/16/S5449 